\title{
Fabrication and Evaluation of Transdermal Microneedles for a Recombinant Human Keratinocyte Growth Factor
}

\author{
Melbha Starlin Chellathurai, Vivien WangTing Ling, Vijayarajkumar Palanirajan
} Faculty of Pharmaceutical Sciences, UCSI University, Kuala Lumpur, Malaysia

\begin{abstract}
Objectives: Microneedles transdermal patches is the combination between hypodermic needles and transdermal patches to overcome the individual limitations of both injections and patches. The objective of this study was to design a minimally invasive biodegradable polymeric recombinant human keratinocyte growth factor (rHuKGF) microneedles array and evaluate the prepared biodegradable microneedles using in-vitro techniques.
\end{abstract}

Materials and Method: Biodegradable polymeric microneedles arrays were fabricated out of poly lactic-co-glycolic acid (PLGA) using micromoulding technique under aseptic conditions and the morphology of the microneedles were characterized using light microscope. Sodium Dodecyl Sulfate-Polyacrylamide Gel Electrophoresis (SDS-PAGE) was used to rule out drug polymer interaction. Standard procedures were used to analyze the prepared microneedles arrays for invitro drug release and microneedles insertion test. Enzyme-linked Immunosorbent Assay (ELISA) was used to quantify rHuKGF.

Results. PLGA polymer was safe to be used for fabrication of rHuKGF microneedles as there was no interaction between drug and polymer. The fabricated rHuKGF microneedles arrays had fully formed microneedles with the height of $600 \mu \mathrm{m}$ and base of $300 \mu \mathrm{m}$. The drug from the microneedles patch was released in-vitro within 30 minutes. The strength of microneedles in the patch was good as it was able to reach the depth of $381 \pm 3.56 \mu \mathrm{m}$ into parafilm without any structural change or fracture.

Conclusion: Microneedles transdermal patches were successfully prepared for rHuKGF and their evaluation suggested excellent quality and uniformity in patch characteristics. This can have potential applications in therapeutic arena offering advantages in terms of reduced dosing frequency, improved patients' compliance and bioavailability.

Keywords: Transdermal drug delivery; Microneedles; Recombinant Human Keratinocyte Growth Factor; Micromoulding; Poly-lactide-co-glycolide.

Melbha Starlin Chellathurai, Faculty of Pharmaceutical Sciences, UCSI University, Kuala Lumpur, Malaysia

melbha.starlin@gmail.com

0000-0002-6496-7080

$+60122309605$

30.10 .2019

02.01 .2020

\section{INTRODUCTION}

Many active drugs cannot be effectively delivered using current drug delivery systems such as injection and pills. 1 uncorrected Microneedles transdermal patches consists of a plurality of micro-projections which helps to pierce the skin upper epidermis far enough to improve the 
delivery of broad range of molecules and nano-particles. Pain-free is promised as it is short enough avoiding the stimulation of nerve fibers. 2 The skin will be restored within one to three days after being treated and no bacterial contamination or long term irritation occurs. To date, microneedles has been used to deliver different molecular weight drugs, bio-therapeutics, vaccines, small molecules and proteins. 3 It is also used in cosmetology as rollers and pens to facilitate the transdermal delivery of peptides and proteins. Compared to solid microneedles made of silicon or metal, the polymeric microneedles has attracted extensive attention because of their excellent biocompatibility, biodegradability and non-toxicity properties. 4 Polymeric microneedles will not leave any sharp biohazard medical wastes after use. Polymers with different degradation profiles and swelling properties allow microneedles to be fabricated with different mechanical properties and performances.5 Poly lactic-co-glycolic acid (PLGA) is one of the most favored FDA approved polymer used in designing biodegradable polymeric microneedles.6 Mucositis is one of the main oncological problems that is caused by high dose cytotoxic cancer chemotherapy or radiotherapy in hematological malignancies patient. It is defined as inflammatory or ulcerative lesion on the mucous membranes lining the entire gastrointestinal tract from the mouth to the anus.7 According to the clinical practice guidelines developed by the Mucositis Study Group of the Multinational Association for Supportive Care in Cancer and the International Society of Oral Oncology (MASCC/ISOO), recombinant Human Keratinocyte Growth Factor (rHuKGF) is recommended for the prevention and treatment of oral mucositis.8 United States Food and Drug Administration (FDA) also approved the utilization of rHuKGF to treat oral mucositis in hematologic malignancies patients who are receiving myeloablative radiochemotherapy with autologous haematopoietic stem cell support.9 Endogeneous Keratinocyte Growth Factor (KGF) is a $28 \mathrm{kDa}$ protein produced naturally in the body by dermal fibroblasts within the skin, lamina propria cells of the intestines and most importantly, mesenchymal cells which is located near to the internal organs. Its epithelial cell proliferative properties help to maintain the epithelial integrity.10 The specificity of KGF on epithelial cells is due to the restricted action only on KGF receptors which is present on the epithelial cells and is absent on the cells of hematopoietic origin.11, 12 The rHuKGF is a recombinant N-terminal truncated form of human KGF, prepared from Escherichia coli using recombinant DNA technology. The molecular weight of $\mathrm{rHuKGF}$ is just $16.2 \mathrm{kDa}$ which is smaller as compared to endogenous KGF due to the removal of first $23 \mathrm{~N}$-terminal amino acids with an elimination half-life (t1/2) of 4.5 hours. 13 It has the similar biological activity as the native protein but with higher stability. 14 Currently $60 \mu \mathrm{g} / \mathrm{kg} /$ day of rHuKGF is administered intravenously daily for 3 consecutive days before and 3 consecutive days after receiving the chemotherapy. The drug is commonly dosed in hospital setting which means that patients has to be hospitalized for a week in order to receive the injection and chemotherapy treatment.15, 16, 17 Because of the inconveniences, pain and economic burden due to hospital charges, patients' compliance will be highly affecte requiring for an alternative route of administration. In our previous research, we had developed chitosan nanoparticles and $\beta$-cyclodextrin based delivery systems

to deliver HuKGF.9, 10, 13 Stability issues due to their complex nature makes protein the difficult drug candidate for transdermal delivery. Therefore, alternative route such as transdermal microneedles is designed to solve the limitations of current parenteral route.

\section{MATERIALS AND METHOD Materials}

Recombinant Human Keratinocyte Growth Factor (rHuKGF) (Sigma Aldrich, USA), Chlorotrimethylsilane, Poly(D, L-lactide-co-glycolic acid) with ratio 75:25 and Polyethylene Glycol 400 were purchased from Sigma-Aldrich (M) Sdn. Bhd., Malaysia. PDMS microneedles mould with $11 \times 11$ arrays (Blueacre Technology, Ireland), polyvinyl Alcohol 
and polyvinyl Pyrrolidone (Chemiz (M) Sdn. Bhd., Malaysia), and ELISA Kit ab183362KGF-FGF-7 (abcam, USA) were used.

\section{Pre-formulation Studies}

\section{Drug-polymer Interaction Studies using SDS-PAGE}

Bio-rad brand SDS-PAGE electrophoresis system was used. Denaturing SDS-PAGE was performed by mixing $10 \mu \mathrm{L}$ of diluted rHuKGF sample (20 ng of protein) with $10 \mu \mathrm{L}$ of sample loading buffer and heated in boiling water for 5 to 10 minutes. Samples and Thermo Scientific ${ }^{\mathrm{TM}}$ Spectra ${ }^{\mathrm{TM}}$ Multicolour Broad Range Protein Ladder were then loaded into precast $12 \%$ Tris-Glycine $1.0 \mathrm{~mm}$ minigels according to Figure 1. Electrophoresis was then performed at room temperature for approximately 45 minutes with a constant voltage of 120 $\mathrm{V}$ in running buffer until the dye front reached the end of the $60 \mathrm{~mm}$ gel. After that, the gel was taken out and washed for three times, 5 minutes each in ultra-pure water. Gel was then stained using Bio-Safe ${ }^{\mathrm{TM}}$ Coomassie Stain for an hour. ${ }^{18}$ De-stain process was required by agitating the gel in $50 \mathrm{~mL}$ of distilled water for minimum 30 minutes. Lastly, the gel was imaged using GS800 Calibrated Densitometer. ${ }^{19}$

\section{Preparation of Backing Membrane}

Film casting method was used to fabricate films and Table 1 shows the formula and composition for the different types of formulated patches. ${ }^{20}$ Petri dishes were first treated with $0.2 \mathrm{~mL}$ of physically mixed liquid paraffin : dichloromethane $(1: 10)$ mixture to facilitate the removal of films from the dishes. The polymer solutions in distilled water were prepared at a concentration of $10 \%$ by dissolving dried powder samples of PVA and PVP at $100^{\circ} \mathrm{C}$ and at room temperature, respectively, followed by vigorous stirring until a clear gel was formed. ${ }^{21}$ Varying proportion of PVP and PVA polymer solutions and different percentage of plasticizer were then mixed well to form a final volume of $150 \mathrm{~mL}$. Bubbles were removed by centrifugation. The resultant solutions were poured onto the petri dishes and dried under ambient conditions for 24 hours in Gelman Sciences microessentials class 100 laminar flow work station. The dried cast films were then detached from the petri dish and wrapped in an aluminum foil for further use. Evaluation tests were carried out on the next day and the formulation with best characteristies was chosen to be used in fabricating microneedles arrays.

\section{Evaluation of Backing Membrane}

Commercialized product Kefentech (ketoprofen plasters) was chosen as the reference.

\section{Physical Appearance}

All the prepared patches were visually inspected for the colour and smoothness.

\section{Thickness of the Film}

The thickness of the films was measured using electronic digital micrometer screw gauge at three different places. ${ }^{22}$ Average values and standard deviation values of the three readings were calculated for each prepared films.

\section{Tensile Strength of the Film and Percentage Elongation Break Test}

Three pieces of film strips $(4 \mathrm{~cm} \times 2 \mathrm{~cm})$ from each formulation were cut evenly and the tensile strength and percentage elongation at break were evaluated using the tensiometer. Tensiometer consists of two load cell grips with the lower one fixed while upper one was movable. Film strip was fixed between these cell grips and the force was gradually applied until the film gets broken. ${ }^{23}$ The tensile strength and percentage of elongation at break was read directly from the dial reading.

\section{Preparation of Medicated Polymeric Microneedles Arrays}

First, the polydimethylsiloxane (PDMS) mould was treated with $0.15 \mathrm{~mL}$ of chlorotrimethylsilane and air dried to facilitate the release of replicated PLGA microneedles 
array from the PDMS mould. Different formulations with different concentration of PLGA polymer solution as shown in Table 2 were used to fabricate the microneedles.

PLGA was accurately weighted and dissolved fully in acetone and finally made up to 1 $\mathrm{mL}$ and was passed through $0.22 \mu \mathrm{m}$ membrane filter to remove the contaminants. After that, $1 \mu \mathrm{L}$ of $\mathrm{rHuKGF}(5 \mu \mathrm{g} / \mathrm{mL})$ was pipetted and incorporated into the molten polymer solution. The amount of $300 \mathrm{uL}$ drug-polymer mixture was then cast into the PDMS microneedle mould using micropipette. Filled PDMS mould was gently placed in the centrifuge and centrifuged at $2000 \mathrm{rpm}$ for 20 minutes for entrapment of drug-polymer mixture into microneedles array cavity in the PDMS mould. The temperature was fixed at $37^{\circ} \mathrm{C}$ during the moulding process. The microneedles were then dried for 24 hours under ambient conditions in a controlled air environment under class 100 laminar flow work station.

After drying of the microneedles, a polymer blend (PVP : PVA) devoid of drug was cast onto the mould. The best formulation of the polymer blend (PVP: PVA in the ratio 4:6 with plasticizer $30 \%$ ) that we concluded from the previous backing membrane evaluation was used. The polymer solution was poured onto the mould and the whole device was air-dried at room temperature under laminar flow hood or freeze-dried overnight. After drying, the dried replicated PLGA microneedles connected to the polymer blend was released from the PDMS mould. Optical images of the microneedle arrays were obtained using light microscope. ${ }^{24,25}$

\section{Evaluation of Polymeric Microneedles}

Morphological Characterization of Polymeric Microneedles Arrays

A Zeiss Axio Vert. A1 inverted microscope (Carl Zeiss, Germany) equipped with HBO 50W mercury vapor lamp and exciter/emitter filter combinations was used for physical characterization of polymeric microneedles. Zen 2012 so fiware (Blue edition) was used for image processing and analysing. Different image sizes were captured and visualized at $5 \mathrm{x}$, 10x and 20x magnifications with different angle of viewing. Three sides of observation carried out: (A) Top view of the microneedles patch (B) Cross section view of the microneedles patch and (C) $70^{\circ}$ view of the microneedles patch. ${ }^{26}$

\section{Microneedles Insertion Test using Light Microscopy}

An eight-layer folded parafilm sheet was used. Thickness of the parafilm sheet was measured at three different places using electronic digital micrometer screw gauge. Microneedles array was first inserted with $20 \mathrm{~N}$ into the parafilm sheet for 30 seconds and removed. The parafilm sheets were then unfolded and number of layers containing holes were counted using light microscope. ${ }^{27}$

\section{In-Vitro Release Test in Saline}

Drug release from microneedles loaded with rHuKGF was determined by using a modified dissolution method. The microneedles array was first pressed against a layer of parafilm in order to let only needles exposed. After insertion, the full penetration of microneedles was confirmed by observing under the light microscope. The parafilm with microneedles was then attached to the bottom of a hollow glass tube in which backing film faced inside the tube while microneedles tips exposed outside. The bottom of hollow glass tube was immersed into freshly prepared $150 \mathrm{~mL} \mathrm{pH} 6.2$ phosphate buffer saline (PBS). The PBS solution was magnetically stirred at $30 \mathrm{rpm}$ and maintained at $37^{\circ} \mathrm{C}$ throughout the test period. Periodically, $100 \mu \mathrm{L}$ aliquot of PBS was sampled and immediately replaced with fresh PBS. The concentration of drug was analysed using ELISA kit and the results were compared with the calibration curve of rHuKGF.

\section{Statistical Analysis}

The results were expressed as mean \pm standard deviation (S.D.) with $n=3$. Simple regression analysis ${ }^{26}$ was performed using GraphPad Prism 7 software. 


\section{RESULT AND DISCUSSION}

\section{Drug-polymer Interaction Studies using SDS-PAGE}

The drug-polymer compatibility evaluation was performed using SDS-PAGE for the drug (rHuKGF), PLGA polymer and their physical mixture (1:1) separately. The results clearly indicates the absence of any chemical interaction between drug and polymer and thus confirming that rHuKGF is compatible with the PLGA polymer and could be used for the preparation of the rHuKGF incorporated microneedles transdermal patch. The rHuKGF used in this study was manufactured by Sigma-Aldrich, United States and had a molecular weight of $18.9 \mathrm{kDa}$ which can be proved by Figure 2 as the gel band of rHuKGF located in between gel band representing $15 \mathrm{kDa}$ and $25 \mathrm{kDa}$. Figure 2(A) shows the gel band of Spectra Multicolor Broad Range Protein Ladder corresponding to its representative molecular weight. The gel band for PLGA polymer is not prominent, due to the specificity of Coomassie stain that could only be used to stain and visualize the proteins. From the resulting bands, there were no interaction observed between PLGA polymer and rHuKGF as the band for the drugpolymer physical mixture was allocated at the same level as that of the band of drug alone. This means that the gel band shown for the mixture was actually the gel band of rHuKGF, evidencing that the drug had not undergone any structural modification or structural change due to chemical interaction.

\section{Preparation and Characterization of Backing Membrane}

Both PVA and PVP are hydrophilic polymers and was found that the thickness of the film increased when the concentration of PVA is increased. Among Formulations A1 - A3, the thickness of the films fabricated varied from $0.236 \pm 0.004 \mathrm{~mm}$ to $0.335 \pm 0.005 \mathrm{~mm}$ as shown in Table 3.

Non-plasticized patches were physically clean, transparent and has a smooth surface. However, the patches were very fragile and thus addition of plasticizer was necessary to improve the mechanical properties of placebo patches.

Formulation A1 with the thinnest film containing PVA : PVP ratio of $4: 6$ was chosen as the control formulation. Further fabrication of films (Formulation B1 - B3) with fixed PVA : PVP polymer ratio of $4: 6$ but different concentration of plasticizer $(20 \%, 25 \%$ and $30 \% \mathrm{w} / \mathrm{w}$ of total polymer) was carried out and the films were evaluated for physical appearance, thickness of the film, tensile strength and percentage elongation break of the film.

The results of mechanical properties of films including tensile strength and percentage elongation at break were shown in Table 4. Commercialized product, Kefentech was chosen as the reference that has $51.448 \pm 8.095 \mathrm{mPa}$ tensile strength and $266.1 \pm 7.411 \%$ of elongation at break. As shown in Table 4, film fabricated from Formulation B3 which contains $30 \% \mathrm{w} / \mathrm{w}$ of plasticizer had the best characteristics as it is the thinnest and had better tensile strength and elongation properties. Its thickness was found to be $0.117 \pm 0.004 \mathrm{~mm}$, tensile strength of $61.362 \pm 3.376 \mathrm{mPa}$ and $288.500 \pm 11.653 \%$ elongation at break. Besides, the results showed that the thickness of patches were uniform as it was evidenced by SD value, which were less than $0.01 \mathrm{~mm}$. Therefore, Formulation B3 was chosen as the model backing membrane for further fabrication of microneedles transdermal patches.

Upon addition of plasticizer, flexibilities of polymer macromolecules or macromolecular segments increases as a result of loosening of tightened intermolecular forces. ${ }^{28}$ This study indicated lower concentrations of plasticizer were found to give rigid and brittle patches whereas higher concentrations gave soft patches. Plasticizer at a concentration of $30 \% \mathrm{w} / \mathrm{v}$ was found to give good flexible patches and gets easily removed from the glass surface without any brittle fracture. All the films formed were transparent, flexible, non-sticky and had a smooth surface. This can ensure that the films will maintain a smooth and uniform surface when applied onto the skin. 
The tensile strength and percentage elongation value of the fabricated formulations depicts that the flexibility increased as the concentration of plasticizer was increased. Sufficient mechanical strength and elongation properties of the backing membrane are important to ease the removal of microneedles transdermal patch from the skin and also help in avoiding the tearing of film while removing.

\section{Preparation and Evaluation of Medicated Microneedles Patches}

PDMS is commonly used to prepare micro-mould micro-devices because it is chemically inert, non-hygroscopic with good thermal stability and is mechanically durable ${ }^{29}$. In this study, PDMS micro-mould was used to fabricate microneedles using micro-moulding technique. Preparation of polymer solution is an important step in the fabrication of microneedles. Each batch of microneedles patches were fabricated using a fresh drug-loaded polymer solution and was stirred well to get uniform dispersion of drug in the solution. However, many bubbles produced in the process of long stirring and might adversely affec the casting process and microneedles shape. The presence of bubbles also might decrease the mechanical strength of the microneedles, which could be a limitation in some situations. Therefore, an optimal centrifugal force and time duration for centrifugation were investigated and were fixed at $2000 \mathrm{rpm}$ for 20 minutes, respectively. Bubbles were success fully removed after centrifugation.

From the results shown in Figure 3, it could be concluded that Formulation M2 with 9\% w/v PLGA polymer solution was the most suitable concentration to fabricate rHuKGF polymeric microneedles as complete and fully formed ones.
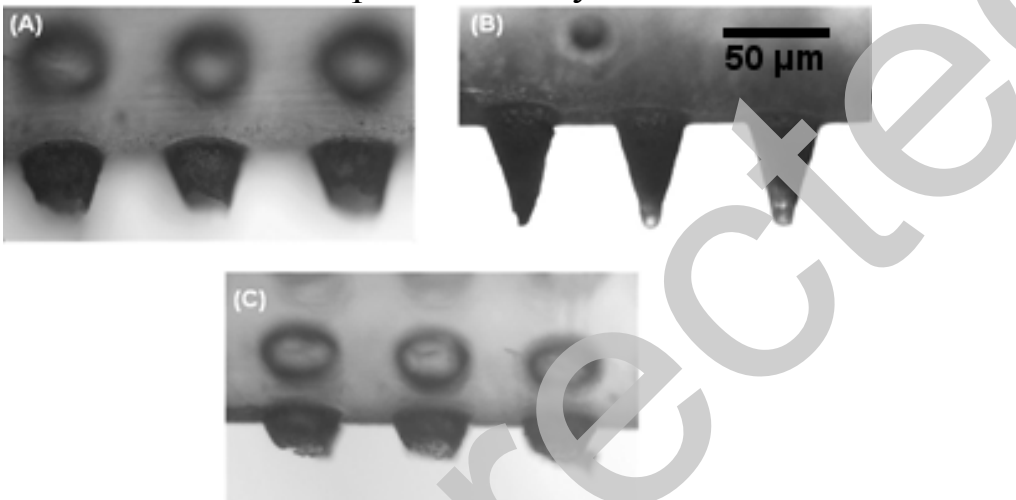

Figure 3 Cross-sectional microscopy images of polymeric microneedles array made from (A) $7 \% \mathrm{w} / \mathrm{v}$ PLGA polymer solution (B) 9\% w/v PLGA polymer solution (C) $11 \% \mathrm{w} / \mathrm{v}$ PLGA polymer solution

The morphology of microneedles was determined at 4x magnification as well as $10 \mathrm{x}$ magnification as shown in Figure 4. Figure 4(D) shows that each needle was $600 \mu \mathrm{m}$ in height and $300 \mu \mathrm{m}$ in base width, and was arranged in a $11 \times 11$ array with $600 \mu \mathrm{m}$ tip-to-tip spacing. Microneedles of this size can penetrate the outer skin barrier and deliver drugs to the epidermis and superficial dermis, where drugs can diffuse rapidly for local delivery to skin or systemic distribution via uptake by dermal capillaries.

The possible reasons for incomplete needle formation by $7 \% \mathrm{w} / \mathrm{v}$ and $11 \% \mathrm{w} / \mathrm{v}$ of PLGA polymer solution could be due to the viscosities of their respective solutions. Solution with low viscosity might get easily spun-off from the opening of the cavity of micro-mould instead of filling into the holes of micro-mould while high viscosity might result in the difficulty to fill in the microneedles mould cavity. 

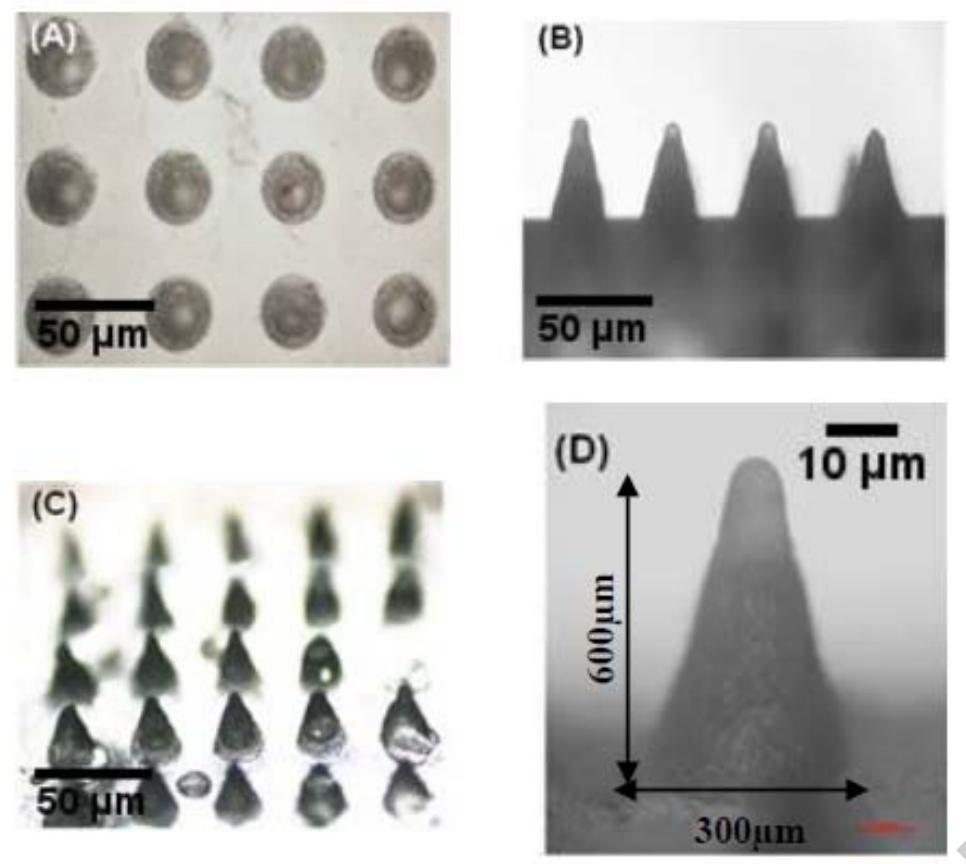

Figure 4 Light microscope images of section of $11 \times 11$ polymeric microneedles array fabricated using Formulation M2: (A) Top View with 4x magnification (B) Cross-Sectional View with $4 x$ magnification (C) $70^{\circ}$ View with $4 x$ magnification (D) Cross Section View with 10x magnification

\section{Microneedles Insertion Test Using Light Microscopy}

Microneedles should have sufficient mechanical strength to be inserted successfully into the skin without failure during insertion. Results of research carried out by Larreneta et. al. ${ }^{27}$ proved that although parafilm presents slightly lower penetration depths than porcine skin, it still could be a promising material to replace biological tissue for insertion studies. Average thickness of a parafilm layer is $127 \pm 3.560 \mu \mathrm{m}$. Third layer of the parafilm sheet can be reached as shown in Figure 5, but holes did not form on third layer which means that the microneedles can correspondingly reach insertion depths between $254 \pm 3.560 \mu \mathrm{m}$ and $381 \pm$ $3.560 \mu \mathrm{m}$.

The average thickness of stratum corneum and epidermis is between 0.01 to $0.02 \mathrm{~mm}$ and 0.1 $\mathrm{mm}$ respectively. Therefore, the results proved that $\mathrm{rHuKGF}$ microneedles can successfully overcome the barrier of stratum corneum and could reach the dermis layer of skin for drug release. Blood vessels are mostly on the lower part of dermis and would not get punctured as the needles penetrate only in the upper layers of the skin. Besides, the mechanical strength of microneedles was good enough as it did not undergo structural change or fracture inside the parafilm sheet after insertion test. Thus, safety of rHuKGF polymeric microneedles is promised. 

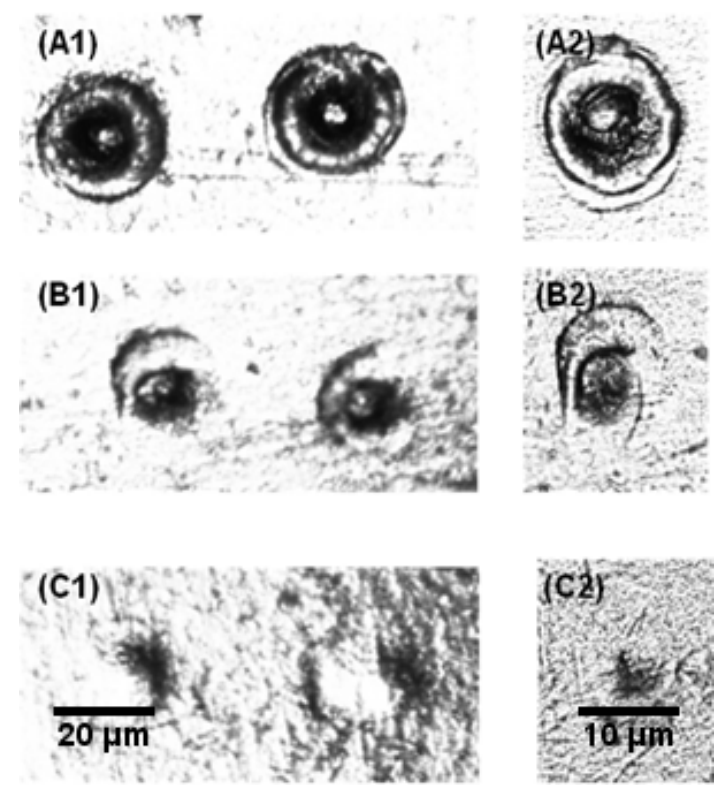

Figure 5 Microscopic observations of holes left on the different layers of parafilm sheet (A) First layer (B) Second layer (C) Third layer with (1) 10x magnification and (2) 20x magnification

\section{In-Vitro Release Test}

Microneedles loaded with $\mathrm{rHuKGF}$ released their contents efficiently within 30 minutes upon incubation in $150 \mathrm{~mL}$ phosphate buffer saline solution with $\mathrm{pH}$ 6.2. Figure 6 showed that $96.67 \%$ of rHuKGF can be successfully released within 15 minutes and $100 \%$ of drug release was obtained within 30 minutes. This rapid release might be due to the burst effect of PVP and the solubility of polymer in the solution. Controlled release was not achieved during the release study that might be due to fabrication methodology.

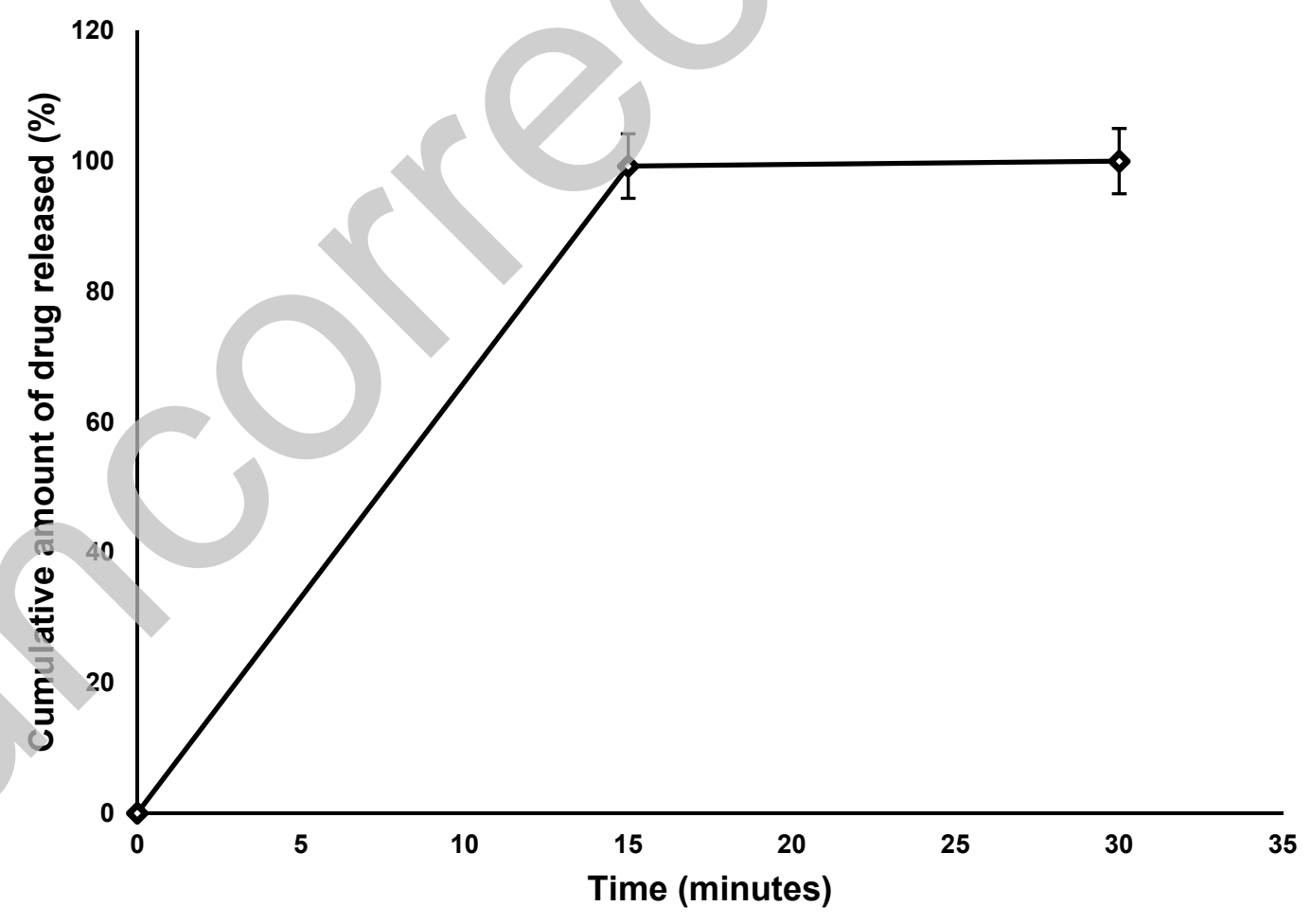

Figure 6 Graph of the Cumulative Release of rHuKGF into Phosphate Buffer Saline Solution at Different Timescale 
As in research done by Park et. al., ${ }^{30}$ additional steps were added before directly encapsulating the drug within microneedles in order to achieve controlled release. The author has mentioned that for controlled release, double encapsulation is necessary in which drug needs to be encapsulated either within carboxymethylcellulose (CMC) or poly-L-lactide (PLA) firstly before getting encapsulated within the microneedles. Although controlled release was not achieved, the results could prove that delivery of rHuKGF by using microneedles technique is successful. Further directions can be focused on the fabrication of controlled release rHuKGF microneedles by using double encapsulating method in order to solve the frequent administration of drugs before and after the chemotherapy.

Theoretically, drug release from microneedles is facilitated either by the drug diffusion through polymer or by the degradation of the polymer. Diffusion is the major path ay of diug release in most controlled release devices and is strongly influenced by polymer matrix, as the motion of a small molecule is restricted by the three dimensional network of polymer chains. In diffusion-controlled release, the molecular size and weight play an important role. However, in case of a dermal application, the surface of the microneedles that pierced into the skin will determine how fast small molecules diffuse from the microneedle arrays into the skin. There are several product-related factors that determine the rate of drug delivery. These factors include the solubility and concentration of the drug molecule, the thickness of the back plate, and the properties of the microneedles array itself such as length, sharpness, porosity, strength, surface area and density ${ }^{31}$. However, the rate of drug delivery is also dependent on variables more difficult to control such as the quality of the penetration, the manner of microneedle application and on the type of skin. To gain more insight into the usability of the polymeric microneedles for drug release into the skin, in-vivo diffusion studies should be performed.

\section{CONCLUSION}

The present study demonstrates polymeric microneedles for transdermal delivery of rHuKGF can be developed using PDMS micro-moulding method. This polymeric microneedle arrays can be fabricated at large scale at low cost. Polymeric microneedles may provide advantages that overcome the limitations of silic on and metal microneedles. Many polymer materials are inexpensive, mechanically strong, and has been used to fabricate medical devices. PLGA polymer fabricated microneedles in this research possessed good mechanical strength and could withstand high forces as it did not get fractured after getting inserted into parafilm sheet which makes the microneedles safe for patients. No interactions were found between rHuKGF and PLGA polymer and the drug could successfully get released from microneedles in-vitro. However, some improvement in reproduction of small scale features such as microneedle tips may be possible. Further investigation such as in-vivo diffusion studies and fabrication of controlled release rHuKGF microneedles for days can be performed.

\section{CONFLICT OF INTEREST}

The authors deciare that there is no conflict of interest regarding the publication of this paper.

\section{ACKNOWLEDGEMENTS}

This project (Reference Number: FRGS/2/2014/SKK02/UCSI/02/1) is supported by

Department of Higher Education, Ministry of Education Malaysia, under the Fundamental

Research Grant Scheme (FRGS) Malaysia and Pioneer Scientists Incentive Fund (PSIF) under Centre of Excellence in Research, Value Innovation and Entrepreneurship (CERVIE), UCSI University, Malaysia (Project Code: Proj-In-FPS-013).

\section{REFERENCES}

1. Larrañeta, E., Lutton, R. E. M., Woolfson, A. D. \& Donnelly, R. F. Microneedle arrays as transdermal and intradermal drug delivery systems: Materials science, manufacture and commercial development. Materials Science and Engineering R: Reports. (2016); 104:1- 
32.

2. Ma, G. \& Wu, C. Microneedle, bio-microneedle and bio-inspired microneedle: A review. Journal of Controlled Release. 2017; 251: 11-23.

3. Kim YC, Park JH, P. M. Microneedles for drug and vaccine delivery. Adv. Drug Deliv. Rev. 2012; 64 (14): 1547-1568.

4. Lee, J. W., Han, M. R., \& Park, J. H. Polymer microneedles for transdermal drug delivery. J. drug targeting,2013; 21 (3): 211-223.

5. Ye, Y., Yu, J., Wen, D., Kahkoska, A. R. \& Gu, Z. Polymeric microneedles for transdermal protein delivery. Advanced Drug Delivery Reviews.2018; 127:106-18.

6. Ulery, B. D., Nair, L. S. \& Laurencin, C. T. Biomedical applications of biodegradable polymers. Journal of Polymer Science, Part B: Polymer Physics. 2011; 3(49): 832-64.

7. Naidu, M. U. R., Ramana, G. V., Rani, P. U., Mohan, I. K., Suman, A., \& Roy, P. Chemotherapy-induced and/or radiation therapy-induced oral mucositis-complicating the treatment of cancer. Neoplasia (New York, NY), 2004; 6(5), 423-431.

8. $\quad$ Keefe, D. M., Schubert, M. M., Elting, L. S., Sonis, S. T., Epstein, J. B., Raber $\square$ Durlacher, J. E., \& Peterson, D. E. Updated clinical practice guidelines for the prevention and treatment of mucositis. Cancer Interdiscip. Int. J. Am. Cancer Soc. 2007; 109(5): 820-31.

9. Kumar, P. V., Maki, A., Abdelmahmoud, M., Takahje, M. L., Wei, Y. S., Tatt, L. M., ... \& Bakar, A. Detection of Formation of Recombinant Human Keratinocyte Growth Factor Loaded Chitosan Nanoparticles Based on its Optical Properties. Curr. Nanosci. 2018;14(2): 127-135.

10. Maki, M. A. A., Kumar, P. V., Cheah, S. C., Siew Wei, Y., Al-Nema, M., Bayazeid, O., \& Majeed, A. B. B. A. Molecular Modeling-Based Delivery System Enhances Everolimus-Induced Apoptosis in Caco-2 Cells. ACS Omega, 2019; 4(5), 8767-8777. 11. Rubin, J. S., Bottaro, D. P., Chedid, M., Miki, T., Ron, D., Cheon, H. G., \& LaRochelle, W. J. Keratinocyte growth factor. Cell Biol. Int. 1995; 19(5), 399-412. 12. Stiff, P. J., Leinonen, M., Kullenberg, T., Rudebeck, M., de Chateau, M., \& Spielberger, R. Long-Term Safety Outcomes in Patients with Hematological Malignancies Undergoing Autologous Hematopoietic Stem Cell Transplantation Treated with Palifermin to Prevent Oral Mucositis. Biol. Blood Marrow Transplant. 2016; 22(1): 164-9.

13. Kumar, P. V., Maki, M. A. A., Wei, Y. S., Tatt, L. M., Elumalai, M., Cheah, S. C., \& Majeed, A. B. B. A.. Rabbit as an Animal Model for Pharmacokinetics Studies of Enteric Capsule Contains Recombinant Human Keratinocyte Growth Factor-Loaded Chitosan Nanoparticles. Curr. Clin. Pharmacol. 2018; 14, 1-9.

14. Braun, S., Hanselmann, C., Gassmann, M. G., auf dem Keller, U., Born-Berclaz, C., Chan, K., ... \& Werner, S. Nrf2 Transcription Factor, a Novel Target of Keratinocyte Growth Factor Action Which Regulates Gene Expression and Inflammation in the Healing Skin Wound. Mol. Cell. Biol. 2002; 22(15): 5492-505.

15. Blijlevens, N. \& Sonis, S. Palifermin (recombinant keratinocyte growth factor-1): A pleiotropic growth factor with multiple biological activities in preventing chemotherapy- and radiotherapy-induced mucositis. Annals of Oncology 2007; 18(5):817-26.

16. Niscola, P., Scaramucci, L., Giovannini, M., Ales, M., Bondanini, F., Cupelli, L., ... \& Romani, C. Palifermin in the Management of Mucositis in Hematological Malignancies:

Current Evidences and Future Perspectives. Cardiovasc. Hematol. Agents Med. Chem. 2009; 7 (4), 305-312.

17. McDonnell, A. M., \& Lenz, K. L. Palifermin: role in the prevention of chemotherapyand radiation-induced mucositis. Ann. Pharmacother.2007; 41(1), 86-94.

18. ThermoFisher Scientific. Protein gel electrophoresis technical handbook Comprehensive solutions designed to drive your success. Thermo Fisher Scientific 2015; 639. 
19. Lin, E. W., Boehnke, N. \& Maynard, H. D. Protein-polymer conjugation via ligand affinity and photoactivation of glutathione s-transferase. Bioconjug. Chem. 2014; 25(10), 1902-1909.

20. Jadhav, R. T., Kasture, P. V., Gattani, S. G., \& Surana, S. J. Formulation and evaluation of transdermal films of diclofenac sodium. Int. J. Pharm Tech Res. 2009; 1(4), 1507-1511.

21. Cassu, S. N. \& Felisberti, M. I. Poly(vinyl alcohol) and poly(vinyl pyrrolidone) blends: Miscibility, microheterogeneity and free volume change. Polymer (Guildf). 1997; 38(15), 3907-3911.

22. Kumar, J. A., Pullakandam, N., Prabu, S. L., \& Gopal, V. Transdermal drug delivery system: an overview. Int J Pharm Sci Rev Res,. 2010. 3(2), 49-54.

23. Prajapati, S. T., Patel, C. G. \& Patel, C. N. Formulation and Evaluation of Transdermal Patch of Repaglinide. ISRN Pharm. 2011;1-9.

24. Fredenberg, S., Wahlgren, M., Reslow, M., \& Axelsson, A.. T. mechanisms of drug release in poly (lactic-co-glycolic acid)-based drug. The mechanisms of drug release in poly (lactic-co-glycolic acid)-based drug delivery systems - a review. Int. J. Pharm. 2011; 415(12), 34-52.

25. Indermun, S., Luttge, R., Choonara, Y. E., Kumar, P., Du Toit, L. C., Modi, G., \& Pillay, V. Current advances in the fabrication of microneedles for transdermal delivery. Journal of Controlled Release. 2014; 185, 130-138.

26. Demir, Y. K., Akan, Z. \& Kerimoglu, O. Characterization of Polymeric Microneedle Arrays for Transdermal Drug Delivery. PLoS One. 2013; 8(10), e77289.

27. Larrañeta, E., Moore, J., Vicente-Pérez, E. M., González-Vázquez, P., Lutton, R., Woolfson, A. D., \& Donnelly, R. F. A proposed model membrane and test method for microneedle insertion studies. Int. J. Pharm. 2014; 472 (1-2), 65-73.

28. Güngör, S., Erdal, M. S., \& Özsoy, Y, Plasticizers in transdermal drug delivery systems. In Recent advances in plasticizers. IntechOpen. Recent Adv. Plast. IntechOpen. 2012.

29. Park, J. H., Allen, M. G. \& Prausnitz, M. R. Biodegradable polymer microneedles: Fabrication, mechanics and transdermal drug delivery. J. Control. Release. 2005; 104(1), $51-$ 66.

30. Park, J. H., Allen, M. G., \& Prausnitz, M. R. Polymer microneedles for controlledrelease drug delivery. Pharmaceutical research, 2006; 23(5), 1008-1019.

31. Van Der Maaden, K., Sekerdag, E., Jiskoot, W. \& Bouwstra, J. Impact-insertion applicator improves reliability of skin penetration by solid microneedle arrays. AAPS J. 2014; 16(4), 681-684 


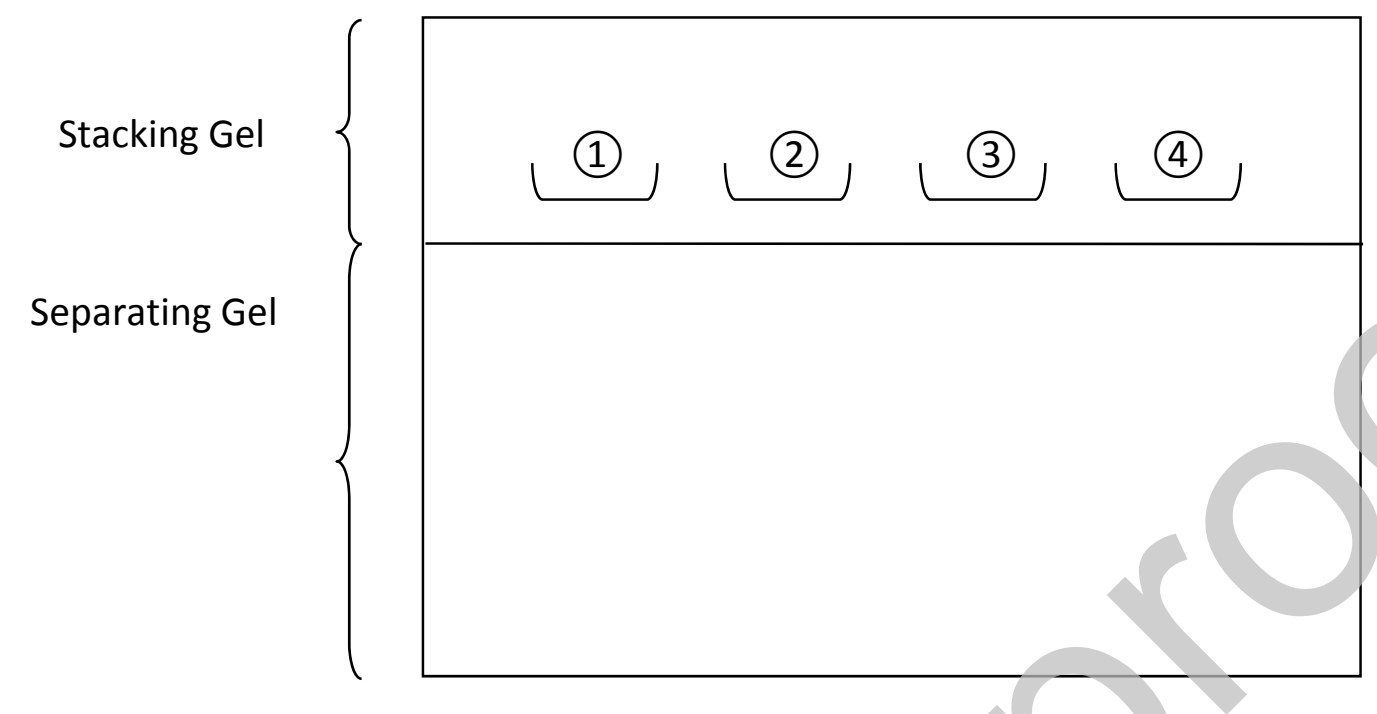

Well (1) : Thermo Scientific ${ }^{\mathrm{TM}}$ Spectra ${ }^{\mathrm{TM}}$ Multicolour Broad Range Protein Ladder

Well (2) : rHuKGF

Well (3) : PLGA Polymer

Well (4) : Physical mixture of rHuKGF and PLGA Polymer

Figure 1: Arrangement of Samples on Gel for Electrophoresis

\begin{tabular}{|c|c|c|c|}
\hline \multirow[t]{2}{*}{$\begin{array}{c}\text { Formulation } \\
\text { Code }\end{array}$} & $\begin{array}{c}\text { Ratio of } \\
\text { Polymer } 10 \% \\
w / v)\end{array}$ & $\begin{array}{l}\text { Plasticizer (\% } \\
\text { w/w of total } \\
\text { polymer) }\end{array}$ & $\begin{array}{l}\text { Solvent / } \\
\text { Patch (mL) }\end{array}$ \\
\hline & PVA: PVP & PEG 400 & $\begin{array}{l}\text { Ultra Pure } \\
\text { Water }\end{array}$ \\
\hline A1 & $4: 6$ & 0 & 150 \\
\hline A2 & $1: 1$ & 0 & 150 \\
\hline A3 & $6: 4$ & 0 & 150 \\
\hline B1 & $4: 6$ & 20 & 150 \\
\hline B2 & $4: 6$ & 25 & 150 \\
\hline B3 & $4: 6$ & 30 & 150 \\
\hline
\end{tabular}


Table 2. Formulation of Medicated Polymeric Microneedles

\begin{tabular}{|l|l|l|}
\hline \multirow{2}{*}{ Formulation Code } & Polymer (\% w/v) & Solvent $(\mathbf{m L})$ \\
\cline { 2 - 3 } & PLGA (75:25) & Acetone \\
\hline M1 & 7 & 1 \\
\hline M2 & 9 & 1 \\
\hline M3 & 11 & 1 \\
\hline
\end{tabular}



A)
B)
C)
D)
E)
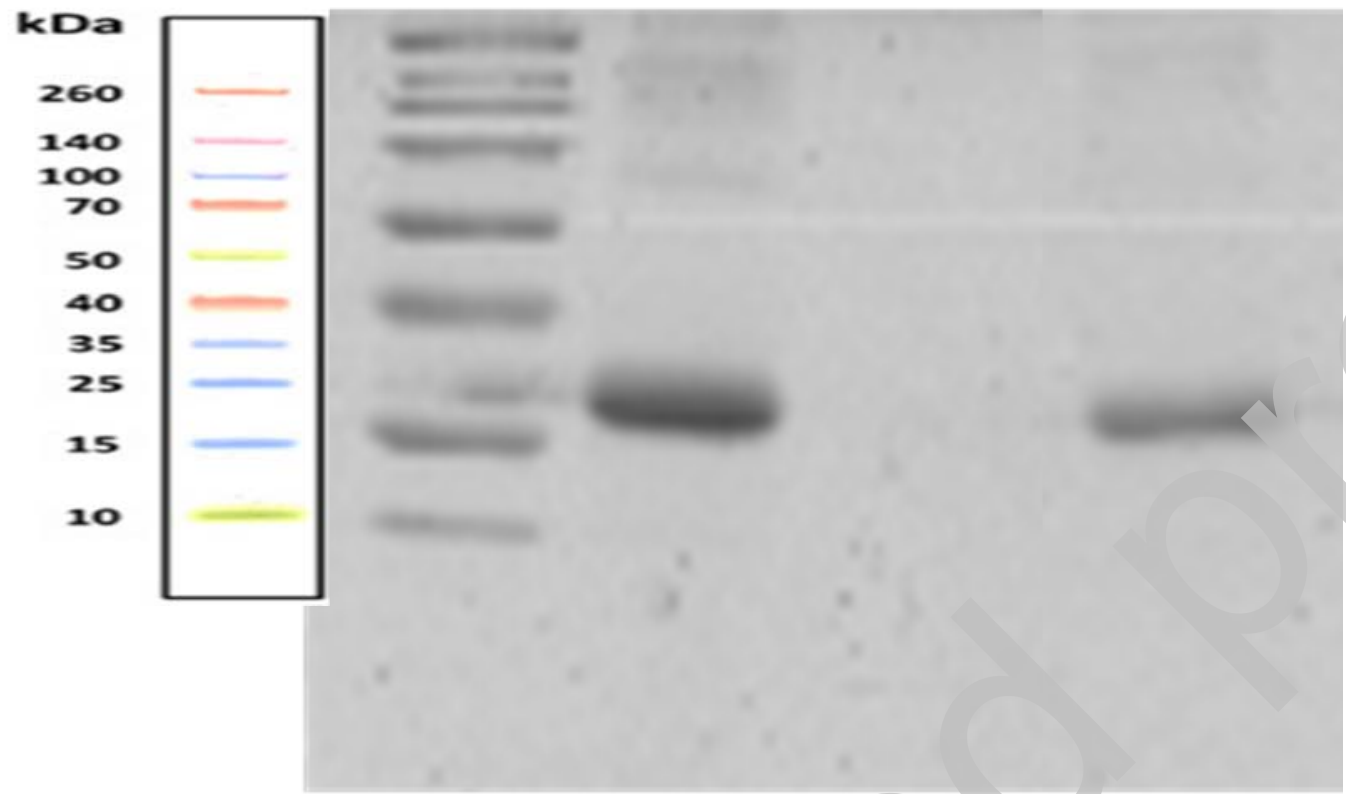

Figure 2 SDS-PAGE Gel band of (A) Spectra Multicolor Broad Range Protein ladder with its molecular weight (B) Spectra Broad Range Protein Ladder (C) rHuKGF (D) PLGA polymer and (E) drug-polymer mixture

Table 3 Thickness of Backing Membrane without Plasticizer

\begin{tabular}{|l|l|l|}
\hline $\begin{array}{l}\text { Formulation } \\
\text { Code }\end{array}$ & $\begin{array}{l}\text { Ratio of Polymer } \mathbf{( 1 0} \% \\
\text { w/v) }\end{array}$ & $\begin{array}{l}\text { Mean Thickness } \\
(\mathbf{m m})\end{array}$ \\
\hline PVA : PVP & \\
\hline A 1 & $4: 6$ & $0.236 \pm 0.0044$ \\
\hline A 2 & $1: 1$ & $0.320 \pm 0.0131$ \\
\hline A 3 & $6: 4$ & $0.335 \pm 0.0045$ \\
\hline
\end{tabular}


Table 4 Physical Characteristics of Films fabricated by Formulation B1 to B3

\begin{tabular}{|c|c|c|c|c|c|}
\hline $\begin{array}{l}\text { Formulation } \\
\text { Code }\end{array}$ & $\begin{array}{l}\text { Ratio of } \\
\text { Polymer } \\
(10 \% \\
\text { w/v) }\end{array}$ & $\begin{array}{l}\text { Plasticizer } \\
(\% \text { w/w of } \\
\text { total } \\
\text { polymer) }\end{array}$ & $\begin{array}{l}\text { Thickness } \\
(\mathrm{mm})\end{array}$ & $\begin{array}{l}\text { Tensile } \\
\text { Strength } \\
\text { (mPa) }\end{array}$ & \% Elongation \\
\hline & $\begin{array}{l}\text { PVA : } \\
\text { PVP }\end{array}$ & PEG 400 & & & \\
\hline B1 & $4: 6$ & 20 & $0.140 \pm 0.0030$ & $24.800 \pm 6.679$ & $244.967 \pm 21.170$ \\
\hline B2 & $4: 6$ & 25 & $0.127 \pm 0.0015$ & $43.356 \pm 6.092$ & $284.767 \pm 12.586$ \\
\hline B3 & $4: 6$ & 30 & $0.117 \pm 0.0036$ & $61.362 \pm 3.376$ & $288.500+1.653$ \\
\hline
\end{tabular}

